\title{
ANTIBACTERIAL AND ANTIBIOFILM ACTIVITIES OF TRANS-CINNAMALDEHYDE NANOEMULSION AGAINST ESCHERICHIA COLI
}

\author{
UTKRISHTA L RAJ, MEGHA GAUTAM, SHWETA DANG, REEMA GABRANI* \\ Department of Biotechnology, Centre for Emerging Diseases, Jaypee Institute of Information Technology, A-10, Sector-62, Noida, \\ Uttar Pradesh, India. Email: reema.gabrani@jiit.ac.in
}

Received: 19 September 2018, Revised and Accepted: 26 October 2018

\section{ABSTRACT}

Objective: Trans-cinnamaldehyde (TC) has shown antimicrobial activity against various microorganisms, but its direct use has some disadvantages such as skin irritation, low bioavailability, and low solubility. The objective of the present work was to develop the oil-in-water nanoemulsions (NEs) of TC to enhance its antimicrobial activity against Escherichia coli.

Methods: The NEs of TC were prepared using triton x-100 and isopropyl alcohol as surfactant and cosurfactant. The developed NE was studied for size, zeta potential, and stability. NEs were evaluated for antimicrobial and antibiofilm activity against $E$. coli as indicator organism. NEs possible mode of action on E. coli was assessed by scanning electron microscopy (SEM).

Results: Stable NEs of TC exhibited a particle size of $210 \mathrm{~nm}$ and were able to inhibit the growth of planktonic as well as biofilm cultures of $E$. coli at $67 \mu \mathrm{g} / \mathrm{ml}$. The ruthenium red staining indicated the inhibition of glycoprotein layer formation in extracellular matrix after treating with NE. TC-NE exhibited substantial decrease in $E$. coli growth as well as its viability at its minimum inhibitory concentration as determined by 3-(4,5-dimethylthiazol2-yl)-2,5-diphenyltetrazolium bromide (MTT) assay. The mode of action of cinnamaldehyde through $\beta$-galactosidase assay on E. coli ML-35p strain indicated that it altered the bacterial cell membrane permeability. SEM results showed the presence of holes on the cell wall of the $E$. coli in the presence of TC-NE.

Conclusions: TC-NEs exhibited enhanced antimicrobial activity and were effective against E. coli biofilm. They also exhibited microbicidal activity and altered E. coli membrane permeability.

Keywords: Growth curve, MTT, Membrane permeability, Ruthenium red, Scanning electron microscopy.

(C) 2019 The Authors. Published by Innovare Academic Sciences Pvt Ltd. This is an open access article under the CC BY license (http://creativecommons. org/licenses/by/4. 0/) DOI: http://dx.doi.org/10.22159/ajpcr.2019.v12i2.27466

\section{INTRODUCTION}

Escherichia coli are the Gram-negative, facultative anaerobic bacteria, generally found in the lower intestine of the warm-blooded organisms. Mostly E. coli strains are harmless; some may cause serious illness such as diarrhea, respiratory illness, uropathogenic infections, and pneumonia [1].

The pathogenicity of $E$. coli is also enhanced with the ability to form a biofilm which significantly increases its virulence. The microorganism forms an aggregate colony as biofilm, which consists of extracellular polymeric substance (EPS), proteins, polysaccharides, and nucleic acids. EPS protects the bacteria attached to the surface from being dispersed and also makes them resistance to the antibiotics, which are one of the reasons for required higher doses of antibiotics to combat the biofilm $[2,3]$.

The excessive use of these antibiotics has led to the development of resistance in microorganisms. It has led to the focus and development of the natural compounds from various plant sources being used as antimicrobial for controlling the growth of the microorganisms. The plant essential oils (EOs) are considered to be an alternative for the existing antimicrobials as they have been proved to contain antimicrobial, antiviral, anti-inflammatory, and antifungal activity $[4,5]$.

Trans-cinnamaldehyde (TC) is one of the main components of the bark of cinnamon plant which is recognized as safe molecule. It is approved by the Food and Drug Administration with no genotoxic or mutagenic effect (TC- 21CFR182.60). The genus Cinnamomum is widely used as a medicine, which is commonly known as cinnamon. Ayurveda identifies it as a medicine for the treatment of common cold, cough, diabetes, fever, flatulence, indigestion, sinusitis, and sore throat [6].

Various studies have proved that TC shows antimicrobial activity against various bacterial strains such as Listeria monocytogenes, Staphylococcus aureus, E. coli, and Salmonella species [7-9]. Cinnamaldehyde was found comparable or better in restricting the growth of Pseudomonas aeruginosa as compared to the standard aminoglycoside antibiotics [10]. However, the use of TC is limited due to the factors such as insolubility in water, the high volatility, low bioavailability, and their role in causing skin irritation [11].

Nanoemulsion (NE) is an important carrier for hydrophobic as well as hydrophilic drugs. Due to their high surface area and kinetic stability, coalescence among the droplets is prevented [12]. It has been shown that the antimicrobial activity of nanoencapsulated EO is enhanced due to its increase in solubility in aqueous phase. Moreover, it acts as a reservoir for bioactive compound which is released gradually over a prolonged time period [13]

In the present work, an o/w NE was formulated with the purpose of encapsulating TC. Antimicrobial and antibiofilm activity of the prepared TC-NE was evaluated against $E$. coli. The structural changes in TC-NE treated $E$. coli cells were explored using scanning electron microscopy (SEM).

\section{MATERIALS AND METHODS}

Materials

TC and dimethyl sulfoxide (DMSO) were purchased from Sigma. Triton $\mathrm{X}-100$, tween-20, tween-80, ethanol, methanol, isopropyl alcohol, 
ruthenium red, MTT, and crystal violet were bought from HiMedia. E. coli 443 was purchased from MTCC - Chandigarh.

\section{Preparation of NE}

The solubility of TC in various surfactants (tween 20 , tween 80 , and triton $\mathrm{X}-100$ ) and cosurfactants (isopropyl alcohol, ethanol, and methanol) was screened to find a suitable NE system. The chosen formulations were homogenized at 10,000 rpm for $10 \mathrm{~min}$ and ultrasonicated (Hielscher, Germany) for 5 min at pulse $10 \mathrm{~s} \mathrm{(ON} \mathrm{and} \mathrm{OFF)} \mathrm{and} \mathrm{amplitude} \mathrm{40 \% .} \mathrm{The}$ NEs so obtained were stored at $4^{\circ} \mathrm{C}$

\section{Characterization of NE}

The prepared NEs were further characterized based on the thermodynamic study, particle size, $\mathrm{pH}$, and viscosity [14]. TC-NE was subjected to thermodynamic study which included heating-cooling cycles (between $4^{\circ} \mathrm{C}$ and $45^{\circ} \mathrm{C}$ ), freeze-thaw cycles (between $-21^{\circ} \mathrm{C}$ and $+25^{\circ} \mathrm{C}$ ), and centrifugation (3500 rpm for $30 \mathrm{~min}$ ). Particle and polydispersity index (PDI) values of TC-NE were analyzed in Zetasizer (100 HS, Malvern instrument, UK), viscosity and $\mathrm{pH}$ were measured using capillary viscometer and $\mathrm{pH}$ meter (Orien 420A+, Electron Corporation).

\section{Antimicrobial assay}

Minimum inhibitory concentration (MIC) of TC-NE and TC was determined by microbroth dilution assay according to the Clinical and Laboratory Standards Institute guidelines [15]. The antimicrobial activity of the $\mathrm{S}_{\text {mix }}$ was also studied and untreated E. coli cells were taken as control. E. coli suspension equivalent to $1 \times 10^{6} \mathrm{CFU} / \mathrm{ml}$ was mixed with various concentrations of TC-NE in microtiter plate assay. The plate was incubated at $37^{\circ} \mathrm{C}$ for $18 \mathrm{~h}$ and the optical density readings were observed using an ELISA plate reader at $595 \mathrm{~nm}$. The assay was performed in triplicates and repeated thrice [16]. The percentage inhibition was calculated as discussed in Sharma et al. [17].

\section{Determination of the bactericidal activity of TC-NE and TC}

The bactericidal activity of the TC-NE was determined by MTT assay on $E$. coli cells. The viability of $E$. coli cells after treatment with TC-NE was also compared with TC and the $\mathrm{S}_{\text {mix }}$. The $E$. coli suspension of cell concentration $1 \times 10^{6} \mathrm{CFU} / \mathrm{ml}$ was added to the 96-well plate along with $\times 2$ MIC, $\times 1$ MIC, and $\times 0.5$ MIC concentrations of TC-NE and TC. The plate was then incubated at $37^{\circ} \mathrm{C}$ for $18 \mathrm{~h}$, and after addition of $5 \mu \mathrm{l}$ of $20 \mathrm{mg} / \mathrm{ml}$ MTT to each well, it was further kept for $3 \mathrm{~h}$. DMSO was added to each well to dissolve the formed formazan crystals and the absorbance was taken using ELISA reader (Biorad, CA, USA) at $570 \mathrm{~nm}$ [18]. The percentage viability was calculated according to the following formula:

$$
\% \text { Viability }=\left[\frac{\text { Absorbance of treated cells }}{\text { Absorbance of untreated cells }}\right] * 100
$$

\section{Growth analysis of $E$. coli when treated with TC-NE and TC}

The growth of $E$. coli in the presence of different concentrations of TC-NE was determined through growth curve. E. coli culture suspension was prepared in similar manner as discussed in previous experiments and incubated with $\times 2 \mathrm{MIC}, \times 1 \mathrm{MIC}$, and $\times 0.5 \mathrm{MIC}$ concentrations of TC-NE. The absorbance of the plate was observed at regular time interval and the results were displayed in the form of graph.

\section{Antibiofilm assay}

The antibiofilm activity of the TC-NE and TC was evaluated both quantitatively and qualitatively. The effect of the various concentrations of TC-NE and TC on biofilm formation of E. coli was quantitatively determined by $\mathrm{CV}$ assay, and the minimum biofilm inhibitory concentration (MBIC) was calculated [17].

In case of qualitative analysis, the biofilm of $E$. coli was grown on coverslips in 6-well plate, alone or in the presence of TC-NE and TC, for $48 \mathrm{~h}$ at $37^{\circ} \mathrm{C}$. After the incubation period, the coverslips were stained with $0.1 \%$ crystal violet stain or ruthenium red separately. These coverslips were then viewed under light microscope [19].

\section{Determination of mode of action of TC-NE}

E. coli cells with (TC-NE) and without treatment were examined under SEM. The culture suspension equivalent to $1 \times 10^{6} \mathrm{CFU} / \mathrm{ml}$ and MIC concentration of TC-NE was incubated for $4-5 \mathrm{~h}$ and then thoroughly washed with PBS buffer 3 times. $1 \mathrm{ml}$ of $0.25 \%$ glutaraldehyde was added and the tubes were incubated overnight at $30^{\circ} \mathrm{C}$. Next day the culture was centrifuged at $8000 \mathrm{rpm}$ for $10 \mathrm{~min}$ and cell pellet was washed with PBS and subsequently with increasing concentration of ethanol (30-90\%) for $5 \mathrm{~min}$ and final 100\% ethanol wash for $1 \mathrm{~h}$. The pellet was viewed under SEM.

\section{RESULTS AND DISCUSSION}

\section{Preparation of NE}

Cinnamaldehyde is an important antimicrobial compound from the cinnamon plant that has been studied for various biological effects. Its direct use is hindered due to the insolubility in water and the allergic reaction on direct contact with the skin. In the present work, NEs are being explored to overcome these problems and also to enhance the antimicrobial activity of the compound. Based on the preliminary solubility studies of TC, surfactant and cosurfactant were chosen for the formulation. TC itself was kept as an oil phase. Depending on solubility of TC, the formulation was optimized using surfactant (30\%) and cosurfactant $(10 \%)$ combinations and end point was kept as transparency of the NE (Table 1). Best results (in terms of transparency) were obtained with three combinations (TC-NE1, TC-NE2, and TC-NE3) which were further characterized for particle size, zeta potential, and PDI (Supplementary File 1). TC-NE2 and TC-NE3 showed particle size and PDI in the desirable range and were further subjected to stability studies. The formulation which showed thermal stability and no phase separation was chosen for further studies (Supplementary File 2).

The chosen formulation (TC-NE2) had triton X-100 (30\%), isopropyl alcohol $(10 \%)$, TC $(800 \mu \mathrm{l})$, and water $(60 \%)$. The $\mathrm{pH}$ of the $\mathrm{NE}$ was found to be 7.00 and the viscosity was calculated to be $9.79 \mathrm{~N} \mathrm{~s} / \mathrm{m}$. The particle size was found to be $210 \mathrm{~nm}$ and the PDI was $=0.465$ which indicated the homogeneity of the sample.

\section{Determination of MIC}

The susceptibility of $E$. coli cells to TC-NE2, TC (pure), and the $\mathrm{S}_{\text {mix }}$ was determined by microtiter plate assay. E. coli was treated with different concentrations and the results showed that the TC-NE2 and TC inhibited the growth of E. coli at MIC values $67 \mu \mathrm{g} / \mathrm{ml}$ and $125 \mu \mathrm{g} / \mathrm{ml}$, whereas corresponding $\mathrm{S}_{\text {mix }}$ did not show any inhibition. This result indicated

Table 1: The outcome for solubility analysis of trans-cinnamaldehyde in various surfactants and cosurfactants

\begin{tabular}{lllll}
\hline Formulation & Surfactant 30\% & Cosurfactant 10\% & Dispersant 60\% & Trans-cinnamaldehyde ( $\boldsymbol{\mu l}$ )/appearance \\
\hline TC-NE1 & Triton X-100 & Ethanol & Water & $800 /$ transparent \\
TC-NE2 & & Isopropyl alcohol & Water & $800 /$ transparent \\
TC-NE3 & & Methanol & Water & $800 /$ transparent \\
TC-NE4 & Tween-20 & Ethanol & Water & $300 /$ opaque \\
TC-NE5 & & Isopropyl alcohol & Water & $300 /$ opaque \\
TC-NE6 & & Methanol & Water & $200 /$ opaque \\
TC-NE7 & Tween-80 & Ethanol & Water & $200 /$ opaque \\
TC-NE8 & & Isopropyl alcohol & Water & $200 /$ opaque \\
TC-NE9 & & Wethanol & Water & $200 /$ opaque \\
\hline
\end{tabular}


that the compound TC/NE-TC was solely responsible for inhibiting the microbial growth. The results also showed enhanced antimicrobial activity of the nanoencapsulated TC with lower MIC value as compared to pure TC.

Determination of bactericidal activity of TC-NE and TC

Subsequent to the establishment of antimicrobial activity, nature of potential antibacterial action of TC-NE2 was determined through MTT assay as shown in Fig. 1. MTT in the presence of NAD(P)H-dependent cellular oxidoreductase enzymes is reduced to blue-colored substrate known as formazan which indicates the presence of viable cells. The killing of $E$. coli cells was determined in the presence of TC-NE2 which indicated that TC-NE2 at MIC concentration showed $<2 \%$ viability of $E$. coli cells as compared to $<8 \%$ viability for TC. The mixture containing $\mathrm{S}_{\text {mix }}$ showed $80 \%$ viable cells. This placebo composition was same as that of TC-NE2 without the active compound. Thus, TC-NE2 demonstrated stronger bactericidal activity than TC as illustrated by $E$. coli cell viability data. The encapsulation of the naturally occurring phytochemical in a nanosystem has been proven to be one of the best approaches to improve their antimicrobial potential. This approach not only helps in improving the antimicrobial potential of the phytochemical but also helps in protecting them from environmental factors such as oxygen, light, and $\mathrm{pH}$ by serving as a barrier between the molecule and the environment $[20,21]$.

\section{Growth analysis of $E$. coli when treated with TC-NE}

The effect of TC-NE2 on E. coli cells in time-dependent manner was shown by plotting growth curve in Fig. 2. The results showed a decline in the growth of $E$. coli cells in the presence of $\times 2$ and $\times 1$ MIC concentrations of TC-NE2. The drop in the growth of cells was more pronounced for $\times 2$ concentration as compared to $\times 1$ concentration of TC-NE. The results were compared with untreated E. coli cells which were taken as positive control.

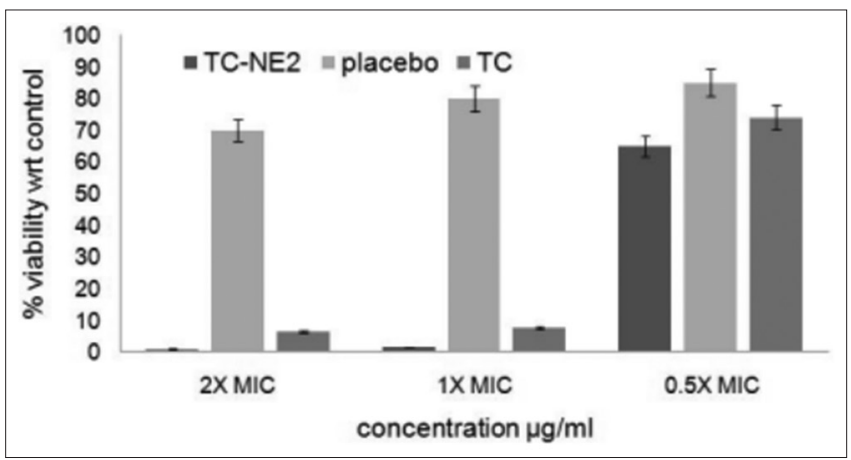

Fig. 1: The percentage viability of the Escherichia coli in the presence of nanoemulsions of trans-cinnamaldehyde (TC-NE2), placebo (P), and TC at respective minimum inhibitory concentration value

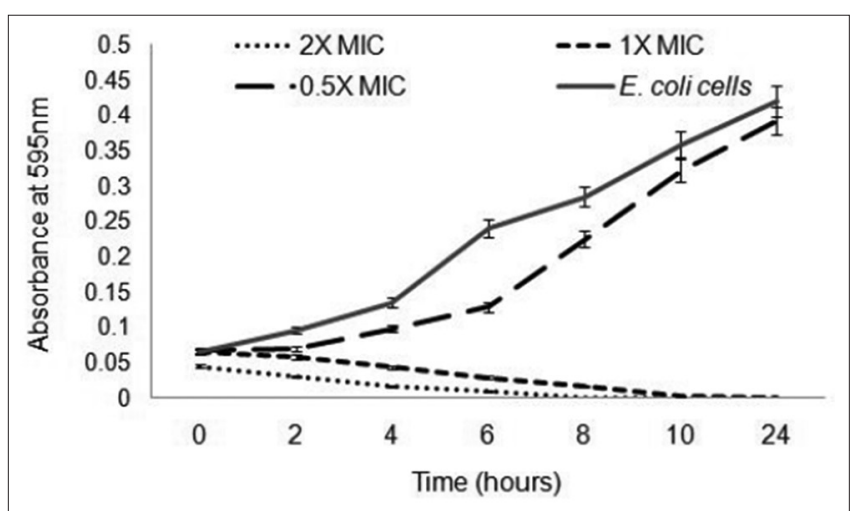

Fig. 2: Growth pattern of Escherichia coli cells exposed to different concentrations of nanoemulsions trans-cinnamaldehyde 2 at different minimum inhibitory concentrations
Various studies have shown that nanoencapsulation of the compound enhances its biological activity. Anwer et al. showed the MIC values of nanoencapsulated clove EO to be ranging from 0.075 to $0.300 \% \mathrm{w} / \mathrm{w}$ as compared to the pure oil which was $0.130-0.500 \% \mathrm{w} / \mathrm{w}$ and, therefore, indicating enhanced antimicrobial activity of encapsulated clove oil [22]. In yet another study, the NE of Thymus daenensis EO was proved to have enhanced antimicrobial activity with MIC value equal to $0.4 \mathrm{mg} / \mathrm{ml}$ which was 10 times more than the activity of pure EO [23]. However, NE of Eucalyptus globulus oil did not show any antimicrobial activity against $P$. aeruginosa probably due to less concentration of oil in the final formulation [24].

\section{Antibiofilm assay}

The effect of TC-NE2, TC, and the $\mathrm{S}_{\text {mix }}$ on the biofilm of E. coli was determined quantitatively and qualitatively. The quantitative analysis by $\mathrm{CV}$ assay showed inhibition of biofilm in the presence of TC-NE2 and TC in dose-dependent manner. MBIC values for TC-NE and TC were found to be $67 \mu \mathrm{g} / \mathrm{ml}$ and $125 \mu \mathrm{g} / \mathrm{ml}$, respectively. $\mathrm{S}_{\text {mix }}$ did not show growth inhibition of $E$. coli cells. The treated and untreated $E$. coli biofilm was also evaluated using crystal violet and ruthenium red staining. The E. coli biofilm, treated with TC-NE2 and stained with crystal violet to determine the cell mass coverage, showed few scattered patches of cells corroborating the quantitative data (Fig. 3). The study also proved that the TC-NE2 was able to inhibit the growth of biofilm at same value as its MIC.

Biofilm of E. coli consists of extrapolymeric substances matrix mainly composed of polysaccharides, proteins, lipids, and nucleic acids [25]. The EPS extent was analyzed by staining with ruthenium red and Fig. 4 indicated much reduced and almost negligible exopolysaccharide extent in TC-NE2 treated biofilm as compared to $\mathrm{S}_{\text {mix }}$ and untreated E. coli samples.

Data pertaining to antibiofilm activity of nanoencapsulated compounds are very limited. Chlorhexidine acetate NE (CNE) was more effective in restricting biofilm of methicillin-resistant $S$. aureus as compared to the unencapsulated compound [26]. Another study indicated that CNE inhibited biofilm formation of cariogenic Streptococcus mutans [27]

Determination of the mode of action of the TC-NE

Surface morphology of the TC-NE2 treated $(67 \mu \mathrm{g} / \mathrm{ml})$ and untreated E. coli cells was analyzed by SEM at $40 \mathrm{~K}$ magnification. The results showed roughened membrane with corrugations and damages at places. Moreover, the disruption in the cell membrane of treated E. coli cells

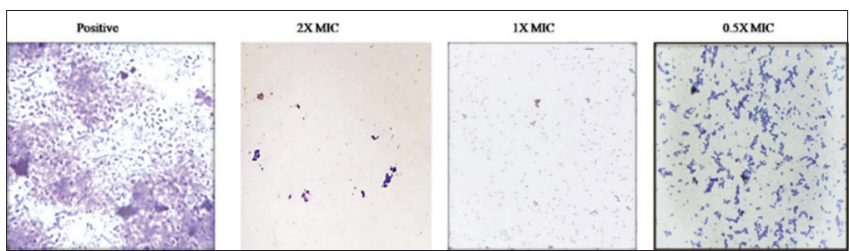

Fig. 3: Crystal violet staining of Escherichia coli biofilm exposed to different minimum inhibitory concentration concentrations of nanoemulsions trans-cinnamaldehyde 2. Positive indicates biofilm of untreated $E$. coli cells

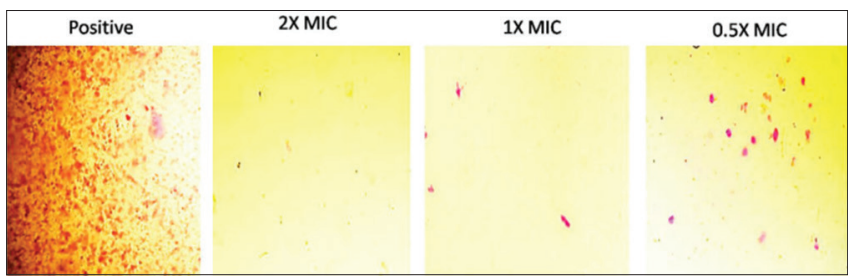

Fig. 4: Exopolysaccharide staining of Escherichia coli biofilm with ruthenium red. Positive denotes untreated $E$. coli biofilm and the other panels indicate the effect of different minimum inhibitory concentration doses of nanoemulsions trans-cinnamaldehyde 2 on $E$. coli biofilm 

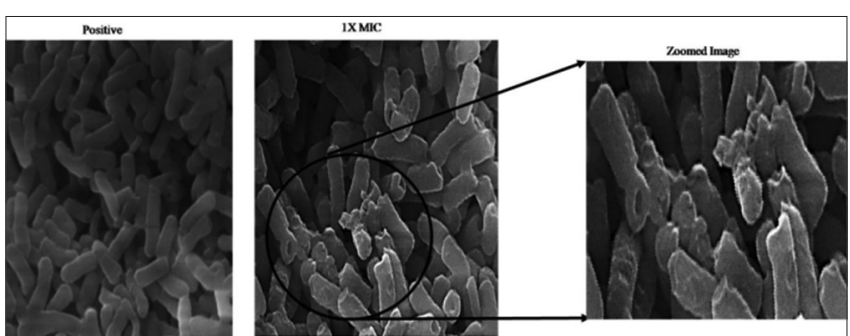

Fig. 5: Scanning electron microscopy images of untreated Escherichia coli cells (positive) and nanoemulsions transcinnamaldehyde 2 treated cells at $\times 1$ minimum inhibitory concentration and its corresponding enlarged image

was evident which could result in the leakage of cell contents resulting in cell death (Fig. 5). Few studies have indicated that nanoencapsulated compound can effectively disrupt the bacterial membrane as possible mode of action. Oil from Neem, one of the ancient medicinal plants when encapsulated in a NE system showed toxicity against pathogenic strain Vibrio vulnificus by disrupting the cell membrane [28]. Song et $a l$. have shown that CNE could damage the cell membrane and result in leakage of ions, protein, and DNA [26].

\section{CONCLUSIONS}

In the present study, we have shown the enhancement of antimicrobial activity of naturally occurring TC when encapsulated in a NE system against E. coli. TC-NE2 was effective in inhibition of growth as well as displayed better bactericidal activity against $E$. coli. Moreover, the TC-NE2 could inhibit the formation of $E$. coli biofilm as depicted by crystal violet and ruthenium red staining. The mechanism of restricting the growth of E. coli could be membrane damage and thus leakage of the contents of cells. Therefore, to conclude, nanoencapsulation of cinnamaldehyde can serve as a potential carrier system and also aid in enhanced antibacterial action.

\section{ACKNOWLEDGMENTS}

The authors thank the Department of Biotechnology, Government of India, for providing financial support to conduct the research work (DBT project No. BT/PR7215/NNT/28/654/2013).

\section{AUTHORS' CONTRIBUTION}

Utkrishta Hira carried out the experiments and wrote the manuscript with support from all authors. Megha Gautam contributed in data analysis and writing of manuscript with input from all authors. Dr. Shweta Dang contributed in designing the study and data analysis and also helped in supervision of project. Dr. Reema Gabrani supervised the project. She conceived and designed the study, its analysis and gave critical feedback in correcting the manuscript.

\section{CONFLICTS OF INTEREST}

There are no conflicts of interest to declare.

\section{REFERENCES}

1. G,Croxen MA, Finlay BB. Molecular mechanisms of Escherichia coli pathogenicity. Nat Rev Microbiol 2010;8:26-38.

2. Ito A, Taniuchi A, May T, Kawata K, Okabe S. Increased antibiotic resistance of Escherichia coli in mature biofilms. Appl Environ Microbiol 2009;75:4093-100.

3. Sabir N, Ikram A, Zaman G, Satti L, Gardezi A, Ahmed A, et al. Bacterial biofilm-based catheter-associated urinary tract infections: Causative pathogens and antibiotic resistance. Am J Infect Control 2017;45:1101-5.

4. Chandra H, Bishnoi P, Yadav A, Patni B, Mishra AP, Nautiyal AR, et al. Antimicrobial resistance and the alternative resources with special emphasis on plant-based antimicrobials-A review. Plants (Basel) 2017;6:E16.

5. Shah B, Davidson PM, Zhong Q. Nanocapsular dispersion of thymol for enhanced dispersibility and increased antimicrobial effectiveness against Escherichia coli $\mathrm{O} 157: \mathrm{H} 7$ and listeria monocytogenes in model food systems. Appl Environ Microbiol 2012;78:8448-53

6. Balijepalli MK, Ayuba SB, Raghavendra S, Mallikarjuna RP. Cinnamomum genus: A review on its biological activities. Int J Pharm Pharm Sci 2017;9:1-11.

7. Nabavi SF, Di Lorenzo A, Izadi M, Sobarzo-Sánchez E, Daglia M, Nabavi SM, et al. Antibacterial effects of cinnamon: From farm to food, cosmetic and pharmaceutical industries. Nutrients 2015;7:7729-48.

8. Shan B, Cai YZ, Brooks JD, Corke H. Antibacterial properties and major bioactive components of cinnamon stick (Cinnamomum burmannii): Activity against foodborne pathogenic bacteria. J Agric Food Chem 2007;55:5484-90.

9. Sheen S, Huang CY, Ramos R, Chien SY, Scullen OJ, Sommers C, et al. Lethality prediction for Escherichia coli $\mathrm{O} 157: \mathrm{H} 7$ and uropathogenic E. coli in ground chicken treated with high pressure processing and trans-cinnamaldehyde. J Food Sci 2018;83:740-9.

10. Grullon J, Mack JP, Rojtman A. Using essential oils to combat the threat of multi-drug resistance bacteria $P$. aeruginosa. Int J Pharm Pharm Sci 2016;8:180-3

11. Jo YJ, Chun JY, Kwon YJ, Min SG, Hong GP, Choi MJ. Physical and antimicrobial properties of trans-cinnamaldehyde nanoemulsions in water melon juice. LWT Food Sci Technol 2015;60:444-51.

12. Álvarez-Paino M, Muñoz-Bonilla A, Fernández-García M. Antimicrobial polymers in the nano-world. Nanomaterials (Basel) 2017;7:E48

13. Demetzos C. Biophysics and thermodynamics: The scientific building blocks of bio-inspired drug delivery nano systems. AAPS PharmSciTech 2015;16:491-5.

14. Donsì F, Annunziata M, Vincensi M, Ferrari G. Design of nanoemulsion-based delivery systems of natural antimicrobials: Effect of the emulsifier. J Biotechnol 2012;159:342-50.

15. CLSI. Methods for Dilution Antimicrobial Susceptibility Tests for Bacteria that Grow Aerobically; Approved Standard. 10 $10^{\text {th }}$ ed. Wayne PA: Clinical and Laboratory Standards Institute, CLSI Document; 2015. p. M07-A10

16. Quave CL, Plano LR, Pantuso T, Bennett BC. Effects of extracts from Italian medicinal plants on planktonic growth, biofilm formation and adherence of methicillin-resistant Staphylococcus aureus. J Ethnopharmacol 2008; 118:418-28.

17. Sharma G, Sharma S, Sharma P, Chandola D, Dang S, Gupta S, et al. Escherichia coli biofilm: Development and therapeutic strategies. J Appl Microbiol 2016;121:309-19.

18. Wang $H$, Cheng $H$, Wang $F$, Wei $D$, Wang $X$. An improved 3-(4,5-dimethylthiazol-2-yl)-2,5-diphenyl tetrazolium bromide (MTT) reduction assay for evaluating the viability of Escherichia coli cells. J Microbiol Methods 2010;82:330-3.

19. Prouty AM, Schwesinger WH, Gunn JS. Biofilm formation and interaction with the surfaces of gallstones by Salmonella spp. Infect Immun 2002;70:2640-9.

20. Donsì F, Annunziata M, Sessa M, Ferrari G. Nanoencapsulation of essential oils enhance their antimicrobial activity in foods. LWT Food Sci Technol 2011;44:1908-14.

21. Wang S, Su R, Nie S, Sun M, Zhang J, Wu D, et al. Application of nanotechnology in improving bioavailability and bioactivity of dietderived phytochemicals. J Nutr Biochem 2014;25:363-76.

22. Anwer MK, Jamil S, IbnoufEO, Shakeel F. Enhanced antibacterial effects of clove essential oil by nanoemulsion. J Oleo Sci 2014;63:347-54.

23. Moghimi R, Ghaderi L, Rafati H, Aliahmadi A, McClements DJ. Superior antibacterial activity of nanoemulsion of thymus daenensis essential oil against E. Coli. Food Chem 2016;194:410-5.

24. Quatrin PM, Verdi CM, de Souza ME, de Godoi SN, Klein B, Gundel A, et al. Antimicrobial and antibiofilm activities of nanoemulsions containing Eucalyptus globulus oil against Pseudomonas aeruginosa and Candida spp. Microb Pathog 2017;112:230-42.

25. Sharma G, Dang S, Gupta S, Gabrani R. Identification and molecular characterization of bacteria having antimicrobial and antibiofilm activity. Int J Pharm Pharm Sci 2016;8:111-4.

26. Song Z, Sun H, Yang Y, Jing H, Yang L, Tong Y, et al. Enhanced efficacy and anti-biofilm activity of novel nanoemulsions against skin burn wound multi-drug resistant MRSA infections. Nanomedicine 2016;12:1543-55.

27. Li YF, Sun HW, Gao R, Liu KY, Zhang HQ, Fu QH, et al. Inhibited biofilm formation and improved antibacterial activity of a novel nanoemulsion against cariogenic Streptococcus mutans in vitro and in vivo. Int J Nanomedicine 2015;10:447-62.

28. Jerobin J, Makwana P, Suresh Kumar RS, Sundaramoorthy R, Mukherjee A, Chandrasekaran N, et al. Antibacterial activity of neem nanoemulsion and its toxicity assessment on human lymphocytes in vitro. Int J Nanomedicine 2015;10 Suppl 1:77-86. 